\title{
O PSICÓLOGO COMPORTAMENTAL E A UTILIZAÇ̃̃O DE TÉCNICAS E INSTRUMENTOS PSICOLÓGICOS
}

\author{
Katya Luciane de Oliveira \\ Ana Paula Porto Noronha\# \\ Marilda Aparecida Dantas" \\ Erica Machado Santarem ${ }^{\infty}$
}

\begin{abstract}
RESUMO. Esta pesquisa teve como objetivo caracterizar as estratégias, técnicas e instrumentos psicológicos mais conhecidos e utilizados por profissionais cuja abordagem norteadora é a comportamental ou cognitiva. Participaram 35 profissionais de diversos estados brasileiros. Utilizou-se um instrumento composto de duas partes. A primeira delas versou questões que visavam à caracterização dos sujeitos quanto à formação, atuação profissional, abordagem terapêutica, atividades profissionais, estratégias e instrumentos utilizados na avaliação. A segunda consistiu em uma relação contendo 152 instrumentos de avaliação psicológica, na qual os sujeitos tinham que assinalar quais instrumentos eram conhecidos e quais eram utilizados. Os resultados evidenciaram que, em sua maior parte, os profissionais atuam em universidades, utilizam avaliação com fins de diagnóstico e intervenção e utilizam instrumentos psicológicos cuja fundamentação teórica não está em consonância com a abordagem terapêutica adotada.
\end{abstract}

Palavras-chave: avaliação psicológica, testes psicológicos, avaliação comportamental.

\section{THE USE OF PSYCHOLOGICAL TECHNIQUES AND INSTRUMENTS FOR BEHAVIORAL PSYCHOLOGISTS}

\begin{abstract}
This research aims feature the behavioral/cognitive psychology and to identify well-know psychology instruments and list used instruments in the sample. Participated this study 35 psychologists by various brazilian states. The material used in this study has two parts. The first comprised questions about professional acting, therapistic choose, professional activity, strategy and instruments used in the assessment. The second part constitute a setoff psychological assessment instruments and the task assigned to subjects was to check know and used. Results indicated the most of professional are acting in university, they used assessment with to aim diagnosis and intervention and they use psychology instruments, of which theoretical foundation is not in accordance with the therapistic choose.
\end{abstract}

Key words: psychological assessment, psychological tests, behavioral.

\section{A AVALIAÇÃo PSICOLÓGICA}

A avaliação psicológica constitui uma prática relativamente recente na psicologia, visto que se configura como campo de produção a partir da metade do século XX. A avaliação está presente no cotidiano, pois todas as pessoas avaliam e também são avaliadas. Toda forma de avaliação pressupõe um julgamento com base em uma concepção explícita ou implícita. Considerando-se a avaliação

* Mestre em Psicologia pelo Programa de Pós-graduação Stricto-sensu em Psicologia da Universidade São Francisco-Itatiba/SP USF, doutoranda em psicologia, desenvolvimento humano e educação pela Faculdade de Educação da UNICAMP e docente do curso de psicologia da Universidade de Alfenas/MG - UNIFENAS e Universidade São Francisco/ Itatiba SP - USF.

\# Doutora em Psicologia: Ciência e Profissão pela PUC-Campinas e docente do Curso de Psicologia e do Programa de Pósgraduação Stricto-sensu em Psicologia da Universidade São Francisco-Itatiba/SP - USF.

II Pós-graduada em Desenvolvimento do Potencial Humano nas Organizações pela PUC-Campinas, mestre em Psicologia pelo Programa de Pós-graduação Stricto-sensu em Psicologia da Universidade São Francisco-Itatiba/SP-USF e docente do curso de psicologia da Universidade de Três Corações/MG - UNINCOR.

x Doutora em Psicologia pela USP e docente do curso de Psicologia, da Universidade São Francisco/São Paulo - SP - USF. 
não como algo inerente ao senso comum, mas como uma atividade que requer rigor e eficácia, pode-se afirmar que a avaliação psicológica é uma prática profissional importante para o psicólogo, tendo-se em vista que pode fornecer elementos de análise imprescindíveis para a atuação em diferentes campos. A realização de avaliações seguras deveria prescindir do conhecimento e do domínio de instrumentos de coleta de dados, dentre eles o teste psicológico (Anastasi \& Urbina, 2000; Casullo, 1999; Pasquali, 1999, 2001).

Os testes são instrumentos de medida que investigam amostras de comportamento e devem ser capazes de auxiliar na identificação de características de sujeitos. Para tanto, devem ser construídos com base científica e apresentar parâmetros psicométricos que de alguma forma atestem a confiabilidade e a representatividade do construto que está sendo medido. Nesse sentido, um teste bem-construído e padronizado oferece a assistência necessária para a investigação de uma variedade de problemas práticos (Anastasi \& Urbina, 2000).

A literatura internacional apresenta padrões para a construção de testes, sendo o Standards for Educational and Psychological Testing (1999) uma das obras de referência no assunto. O objetivo do material é oferecer ao leitor critérios de avaliação da qualidade dos testes, assim como sobre a prática e uso dos testes. Esse tipo de trabalho tende a solucionar dúvidas relacionadas quer ao uso qquer à construção de instrumentos de medida (American Educational Research Association, American Psychological Association \& National Council on Measurement in Education, 1999).

Outra publicação de destaque, do mesmo nível de importância da anterior, refere-se ao material produzido pelo International Test Commition (ITC, 2001), que assegurou informações valiosas sobre uso, construção e revisão de instrumentos. Os trabalhos colaboraram para o estabelecimento de critérios para a construção de instrumentos com base em normalizações e estudos de padronização.

Todos esses esforços voltadosımelhorar a qualidade dos instrumentos psicológicos são muito importantes para a área de avaliação psicológica. No Brasil diversos pesquisadores têm se dedicado a estudar mais profundamente os instrumentos psicológicos disponíveis no mercado (Noronha, Primi \& Alchieri, 2002). As pesquisas vão desde estudos de padronização ou normatização de instrumentos até aquelas que discorrem sobre a qualidade e comercialização de tais instrumentos. Embora as pesquisas sobre a utilização de testes tenham crescido na última década, Noronha (1999), pesquisando os usos e problemas na utilização dos testes psicológicos, constatou que os psicólogos não costumam utilizar testes psicológicos na sua avaliação.

Outro dado que merece atenção é que a formação na área de ensino de testes é deficiente, como observado por Oliveira, Noronha, Beraldo e Santarem (2003). Muitas vezes o estudante sai da graduação e passa a utilizar diversos instrumentos psicológicos sem o mínimo conhecimento técnico necessário para a manipulação do material, sendo levado a avaliações inadequadas, o que certamente denigre a imagem da profissão.

No que tange aos profissionais que estão no mercado há mais tempo, o ideal seria que houvesse cursos que ensinassem ou aprimorassem seu conhecimento sobre a adequada manipulação dos instrumentos psicológicos, enfatizando quais as propriedades psicométricas que um teste bemconstruído deve possuir. Isso certamente evitaria diversos problemas inerentes à utilização inadequada dos testes psicológicos. Nesse sentido, Almeida (1999) observa que as informações coletadas com os testes psicológicos dependem não somente da qualidade do instrumento, mas também do conhecimento e competência científica de quem o utiliza. Conhecer e saber manipular um instrumento psicológico é algo que merece mais atenção no âmbito psicológico, pois, conforme preconizam Anastasi e Urbina (2000), há a necessidade de se ter um conhecimento básico sobre os testes, visto que servem de fonte de informações para as pessoas tomarem decisões sobre si ou sobre os outros.

\section{A AVALIAÇÃO COMPORTAMENTAL}

A análise comportamental no Brasil iniciou-se em 1961, propriamente na Universidade de São Paulo. Desde então, a teoria foi introduzida nas demais instituições e vem gerando frutos até hoje (Matos, 1998).

A análise comportamental prioriza os relacionamentos funcionais entre fatores de desenvolvimento e de manutenção. Diante deste contexto, os objetivos principais da avaliação clínica são identificar as variáveis controladoras dos comportamentos problemáticos, entender a interação funcional e predizer o comportamento futuro. Salienta-se, ainda, que a avaliação busca verificar as 
hipóteses num processo contínuo de testagem (Lettner, 1998).

Para Rangé (1998), a terapia comportamental apresenta um diferencial no que se refere à ênfase em medidas. Essas medidas resultam da observação de pacientes em entrevistas e de outros registros elaborados pelo psicólogo ou indivíduos treinados por ele, e ainda de instrumentos de medida previamente validados.

Nesse sentido, Godoy (1996) discutiu o processo da avaliação comportamental e especificou três fases principais. A primeira se refere à seleção e descrição dos comportamentos-problema. A segunda é concernente à seleção das técnicas de intervenção que serão aplicadas sobre os comportamentos descritos na fase anterior e a terceira diz respeito à avaliação dos efeitos provocados pela intervenção realizada.

Cunha (1998) discutiu a análise aplicada do comportamento como forma de compreender e predizer o comportamento. Nesse sentido, comportamento se define como qualquer ação do indivíduo que pode ser observada por outra pessoa e descrita cientificamente em termos observáveis e mensuráveis. À luz da teoria comportamental, após a identificação do comportamento a ser modificado, podem-se definir variáveis que se relacionam com um comportamento específico, desta forma pode-se predizer e controlar a ocorrência de um comportamento.

As características que mais fortemente discriminam a avaliação comportamental da não comportamental foram levantadas por Haynes e O' Brien (2000), que as classificaram quanto a seus objetivos, focos, metas, estratégias, inferências, suposições sobre causalidade e propriedades dos comportamentos-problemas. Os autores enfatizaram, dentre outras, variáveis observáveis em oposição a variáveis ou eventos intrapsíquicos da avaliação não comportamental e, também, a busca de relações funcionais envolvendo variáveis de estímulos e de respostas, quando se referiam ao foco das avaliações.

Quanto às estratégias, métodos e inferências, ressaltou-se a preferência da avaliação comportamental por estratégias de medidas de séries temporais de casos únicos em oposição a medidas discretas e normativas. No entanto, estratégias da avaliação idiográfica são freqüentemente combinadas com as da avaliação nomotética, segundo Haynes e $\mathrm{O}$ Brien (2000), principalmente na abordagem comportamental-cognitiva. Procedimentos de observação direta do comportamento-problema no ambiente natural ou análogo são estratégias preferenciais, porém, mais difíceis de serem realizadas no contexto clínico não institucional.

Haynes e O’ Brien (2000) destacaram, por último, a avaliação comportamental em variáveis causais presentes, não só pela maior utilidade clínica dessas variáveis em contraposição às variáveis etiológicas originais, mas também pela coerência com a noção de causalidade sobre o comportamento-problema embasada nos princípios da aprendizagem social e da análise experimental do comportamento.

Diversos autores têm apontado que, além da análise funcional, os testes e inventários também podem ser utilizados como auxiliares na avaliação (Piotrowski \& Lubin, 1990; Haynes \& O’Brien, 2000; Meyer, 2001); mas é preciso ressaltar que tais instrumentos devem ser respaldados na teoria psicométrica de construção de instrumento.

Para Oliveira e cols. (2003) a escolha do instrumento também deve seguir critérios que sejam compatíveis ao referencial teórico da abordagem, não havendo como um terapeuta cuja abordagem é comportamental ou cognitiva utilizar um instrumento cuja fundamentação teórica encontra-se respaldada na teoria tradicional de personalidade.

A consonância entre a abordagem e a escolha dos instrumentos psicológicos não vem sendo considerada na avaliação. Essa falta de coerência teórica vem sendo discutida em diversas pesquisas, como é o caso da de Watkins, Campbell e McGregor (1990), a qual evidenciou que psicólogos comportamentais ou cognitivos utilizam técnicas projetivas gráficas em suas avaliações.

Sob esse aspecto Oliveira e cols. (2003) pesquisaram quais os instrumentos utilizados por estagiários de clínica comportamental e constaram que esses estudantes, futuros profissionais, utilizam inventários de personalidade e técnicas gráficas projetivas para compor sua avaliação. Causa espanto esse fato, visto que a coerência teórica deveria ser ensinada desde a graduação, mas, ao que parece, não é o que acontece. Se esse fato está acontecendo com estudantes que estão prestes a ingressar no mercado de trabalho, acredita-se que também aconteça com profissionais que já estão atuando no mercado.

\section{OBJETIVO}

O estudo tem como objetivo caracterizar as estratégias, técnicas e instrumentos psicológicos mais conhecidos e utilizados por profissionais, cuja 
abordagem que norteia a prática é a comportamental ou cognitiva.

\section{MÉTODO}

\section{Participantes}

Participaram 35 psicólogos de diversos estados e universidades brasileiras, cuja abordagem adotada era comportamental ou cognitiva. A idade variou de 22 a 60 anos, com uma média de 34 anos e nove meses de idade e $D P=10,7$. O gênero feminino constitui a maior parte da amostra $(82,9 \% ; n=29)$ e o masculino representou $17,1 \%(n=6)$.

\section{Instrumentos}

Utilizou-se um instrumento composto de duas partes. A primeira continha questões que visavam à caracterização dos sujeitos quanto à formação, abordagem terapêutica (se adotava a linha cognitiva, a filosofia e metodologia da análise funcional do comportamento ou alguma abordagem eclética, sendo que se o profissional escolhesse essa alternativa havia um espaço pedindo que informasse qual era a abordagem adotada), atividades profissionais, estratégias e instrumentos utilizados na avaliação.

A segunda parte consistiu em uma relação contendo instrumentos de avaliação psicológica específicos. $\mathrm{Na}$ relação estavam presentes 152 instrumentos das seguintes editoras: Cepa, Vetor, Casa do Psicólogo, Edites, Cetepp, Artes Médicas, Editorial Psy, Mestre Jou, Melhoramentos, Entreletras, Gráfica MNJ Ltda, Martins Fontes, 10 testes estrangeiros e 2 testes de editoras não localizadas. Os sujeitos tiveram duas possibilidades de resposta para cada instrumento listado, a saber: 'C' para os instrumentos que conhecia (mas não utilizava) e ' $U$ ' para instrumentos que são por eles utilizados. Caso o instrumento não fosse utilizado ou conhecido, o sujeito não deveria fazer nenhuma anotação.

\section{Procedimento}

A coleta de dados ocorreu em universidades dos estados de Minas Gerais e São Paulo, bem como em profissionais que tinham consultórios particulares nesses estados. Utilizou-se, ainda, o IV Congresso Brasileiro de Psicologia Escolar e Educacional para a coleta dos dados, desse modo a amostra ficou bem diversificada, com profissionais de vários estados e universidades brasileiras. Em todas as ocasiões a aplicação foi individual, salientando-se que o instrumento é auto-aplicável. Os sujeitos que consentiam em participar da pesquisa respondiam o instrumento e em seguida o entregavam às pesquisadoras. Cabe salientar que esta pesquisa foi aprovada pelo comitê de ética da universidade à qual está vinculada.

\section{RESULTADOS}

Após a coleta, os dados foram organizados em planilhas e submetidos à estatística descritiva, de acordo com os objetivos do estudo. A análise foi realizada a partir da freqüência das respostas e das respectivas porcentagens.

A primeira e a segunda questões visaram caracterizar o grau de graduação e a atuação profissional dos psicólogos, com seus respectivos ramos de atividade. Os resultados evidenciaram que $31,4 \% \quad(n=11)$ dos sujeitos são graduados em psicologia; 22,9\% (n=8) são especialistas; $20 \%(n=7)$ possuem mestrado, os doutores tiveram a mesma porcentagem de representação que os mestres (20\%; $n=7)$ e $5,7 \%(n=2)$ responderam que possuem outro nível de graduação.

Para a questão sobre a atuação profissional considerou-se o número total de citações nas categorias de respostas, que foi 64 , observando-se que os profissionais poderiam assinalar mais de uma alternativa de atuação. A porcentagem de citações de profissionais que atuam em consultórios particulares foi de 26,5\% $(n=17)$ e em hospitais $9,4 \%(n=6)$. A porcentagem de citações de atuação em escolas públicas foi de $3,1 \%(n=2)$, as escolas particulares obtiveram a porcentagem de $4,7 \%$ $(n=3)$ e a porcentagem de citações de profissionais que atuam em universidades foi de 32,9\% ( $n=21)$. A atuação em instituições apresentou uma porcentagem de citações de 7,8\% (n=5), e 9,4\% $(n=6)$ foi a porcentagem de citações relativa aos profissionais que atuam em organizações. Apenas $6,2 \%(n=4)$ citaram que têm outro tipo de atuação profissional.

As duas questões subseqüentes versavam sobre a abordagem filosófica e terapêutica adotada (comportamental cognitiva, filosofia e metodologia da análise funcional ou eclética) e inquiriam se o profissional possuía atividade paralela à atuação terapêutica, mas dentro da psicologia.

A porcentagem de profissionais que responderam ser a abordagem norteadora de sua prática a comportamental cognitiva foi de 68,6\% (n=24), e $22,9 \%(n=8)$ correspondiam à porcentagem dos que 
responderam que adotam a filosofia e a metodologia da análise funcional. A abordagem eclética foi escolhida por $8,5 \%(n=3)$ dos profissionais, e se o profissional tivesse assinalado essa alternativa havia uma pergunta que solicitava a indicação de qual era essa abordagem; todavia nenhum profissional respondeu à questão.

No que se refere ao exercício de atividade paralela à atuação terapêutica, a maioria dos profissionais $(51,4 \%$; $n=18$ ) responderam que exercem atividade paralela à atuação terapêutica. A porcentagem de profissionais que responderam não possuir atividade paralela à atuação terapêutica foi de 34,3\% (n=12), e 14,3\% (n=5) não responderam a essa questão.

Constatou-se que a avaliação qualitativa é feita por $91,4 \% \quad(n=32)$ dos profissionais, sendo que $68,6 \% \quad(n=24)$ sempre realizam esse tipo de avaliação, 22,9\% ( $n=8)$ o realizam ocasionalmente e $8,5 \% \quad(n=3)$ não responderam à questão. Já a avaliação quantitativa é realizada por $88,6 \% \quad(n=31)$ dos profissionais. A porcentagem de profissionais que responderam que sempre fazem esse tipo de avaliação foi de $51,4 \% \quad(n=18) ; 34,3 \% \quad(n=12)$ assinalaram a alternativa ocasionalmente; $2,9 \%$ $(n=1)$ assinalaram nunca ter realizado uma avaliação desse tipo e $11,4 \% \quad(n=4)$ dos sujeitos deixaram de responder a essa questão.

A finalidade da avaliação e sua freqüência de utilização também foram questionadas. As alternativas de resposta quanto à frequiência de utilização variaram entre sempre, ocasionalmente e nunca. Os dados podem ser observados na Tabela 1 . Saliente-se que $14,3 \% \quad(n=5)$ dos profissionais responderam que exercem outra atividade profissional.

Tabela 1. Distribuição das Frequiências e Porcentagens Referentes à Finalidade da Avaliação Realizada pelo Profissional $(n=35)$

\begin{tabular}{lcccc}
\hline \multirow{2}{*}{$\begin{array}{l}\text { Finalidade da } \\
\text { Avaliação }\end{array}$} & $\boldsymbol{F ~ e ~ \% ~}$ & $\boldsymbol{F}$ e \% & $\boldsymbol{F}$ e \% & $\boldsymbol{F ~ e ~ \% ~}$ \\
\cline { 2 - 5 } Sempre & Ocasionalmente & Nunca & não respondentes \\
\hline Diagnóstico & 28 & 4 & 0 & 3 \\
& 80 & 11,4 & 0 & 8,6 \\
\hline Intervenção & 23 & 7 & 1 & 4 \\
\hline Avaliação da & 65,7 & 20 & 2,9 & 11,4 \\
intervenção & 20 & 6 & 2 & 7 \\
\hline Avaliação de & 21,1 & 17,1 & 5,7 & 20 \\
Resultados & 60 & 6 & 1 & 7 \\
\hline \multirow{2}{*}{ Encaminhamento } & 13 & 17,1 & 2,9 & 20 \\
\hline Avaliação & 37,1 & 14 & 1 & 7 \\
psicológica & 7 & 40 & 2,9 & 20 \\
\hline
\end{tabular}

Os questionamentos sobre a utilização de estratégias e instrumentos de avaliação pelos profissionais foram divididos em duas partes. A Tabela 2 apresenta os resultados da primeira parte, que contemplou uma questão geral, na qual foi perguntado com qual frequiência (sempre, ocasionalmente e nunca) o profissional utilizava as estratégias e instrumentos listados na relação, quando de uma avaliação.

Tabela 2. Distribuição das frequiências e porcentagens de utilização de estratégias e instrumentos de avaliação $(n=35)$.

\begin{tabular}{|c|c|c|c|c|}
\hline \multirow[b]{2}{*}{ Estratégias e Instrumentos } & $F \mathrm{e} \%$ & $F \mathrm{e} \%$ & $F \mathrm{e} \%$ & $F \mathrm{e} \%$ \\
\hline & Sempre & Ocasionalmente & Nunca & $\begin{array}{c}\text { não } \\
\text { respondentes }\end{array}$ \\
\hline \multirow[t]{2}{*}{ Entrevistas } & 31 & 4 & 0 & 0 \\
\hline & 88,6 & 11,4 & & \\
\hline \multirow[t]{2}{*}{ Questionários } & 15 & 15 & 5 & 0 \\
\hline & 42,8 & 42,8 & 14,3 & \\
\hline \multirow{2}{*}{$\begin{array}{l}\text { Observação por observadores } \\
\text { externos no ambiente natural }\end{array}$} & 19 & 7 & 5 & 4 \\
\hline & 54,3 & 20 & 14,3 & 11,4 \\
\hline \multirow{2}{*}{$\begin{array}{l}\text { Observação por observadores } \\
\text { externos no ambiente controlado }\end{array}$} & 7 & 13 & 10 & 5 \\
\hline & 20 & 37,1 & 28,6 & 14,3 \\
\hline \multirow{2}{*}{$\begin{array}{l}\text { Observação por meio de } \\
\text { instrumentos (ex. gravação de } \\
\text { discussão famíliar) }\end{array}$} & 4 & 9 & 17 & 5 \\
\hline & 11,4 & 25,7 & 48,6 & 14,3 \\
\hline \multirow{2}{*}{$\begin{array}{l}\text { Observação qualitativa durante } \\
\text { entrevistas ou sessões terapêuticas }\end{array}$} & 10 & 10 & 7 & 8 \\
\hline & 28,6 & 28,6 & 20 & 22,8 \\
\hline \multirow[t]{2}{*}{ Observação participante } & 12 & 7 & 9 & 7 \\
\hline & 34,3 & 20 & 25,7 & 20 \\
\hline \multirow{2}{*}{$\begin{array}{l}\text { Registros de auto-observação do } \\
\text { cliente }\end{array}$} & 17 & 8 & 8 & 2 \\
\hline & 48,5 & 22,9 & 22,9 & 5,7 \\
\hline \multirow[t]{2}{*}{ Inventários de personalidade } & 7 & 17 & 7 & 4 \\
\hline & 20 & 48,6 & 20 & 11,4 \\
\hline \multirow{2}{*}{$\begin{array}{l}\text { Escalas de classificação do } \\
\text { comportamento }\end{array}$} & 12 & 14 & 7 & 2 \\
\hline & 34,3 & 40 & 20 & 5,7 \\
\hline \multirow[t]{2}{*}{ Técnicas projetivas gráficas } & 5 & 11 & 10 & 9 \\
\hline & 14,3 & 31,4 & 28,6 & 25,7 \\
\hline \multirow[t]{2}{*}{ Testes psicológicos } & 9 & 18 & 3 & 5 \\
\hline & 25,7 & 51,4 & 8,6 & 14,3 \\
\hline
\end{tabular}

As Tabelas 3 e 4 mostram os resultados relativos à segunda parte, contendo questão que versava sobre a verificação dos instrumentos específicos mais conhecidos e utilizados pelos profissionais. Para essa parte da questão os profissionais deveriam assinalar, na relação dos instrumentos, ' $C$ ' se conhecia o instrumento e ' $U$ ' se o instrumento era utilizado. 
Tabela 3. Distribuição das Freqüências e Porcentagens Referentes aos Instrumentos Psicológicos mais Conhecidos por Psicólogos Comportamentais $(n=35)$

\begin{tabular}{|c|c|c|c|c|c|}
\hline Testes Mais conhecidos & $\boldsymbol{F}$ & $\%$ & Testes Mais conhecidos & $\boldsymbol{F}$ & $\%$ \\
\hline Teste de Apercepção Temática TAT & 23 & 65,7 & Lendo e Escrevendo & 5 & 14,3 \\
\hline Teste Zulliger & 23 & 65,7 & Questionário Desiderativo & 5 & 14,3 \\
\hline WISC III & 23 & 65,7 & Diagnóstico Organizacional & 4 & 11,4 \\
\hline Teste de Apercepção Infantil CAT-H & 20 & 57,1 & Escala de Escolha Profissional & 4 & 11,4 \\
\hline Teste de Rorschach & 20 & 57,1 & Inventário Ilustrado de Interesse GEIST & 4 & 11,4 \\
\hline Escala de Maturidade Mental Columbia & 19 & 54,3 & Questionário Saúde Geral Goldberg QSG & 4 & 11,4 \\
\hline Teste de Apercepção Infantil CAT-A & 19 & 54,3 & Teste de Apercepção para idosos & 4 & 11,4 \\
\hline Teste de Inteligência Não Verbal G36 & 18 & 51,4 & Teste de Maturidade para leitura TML & 4 & 11,4 \\
\hline Dezesseis PF & 17 & 48,6 & Teste Prontidão Emoc.Motoristas TEPEM & 4 & 11,4 \\
\hline Teste das Fábulas & 17 & 48,6 & Teste Diagn. Habilidade Pré-escolar DHP & 4 & 11,4 \\
\hline Bateria CEPA & 17 & 48,6 & Teste Retenção Visual Benton & 4 & 11,4 \\
\hline Cubos de Kohs & 16 & 45,7 & Teste Verbal de Inteligência V-47 & 4 & 11,4 \\
\hline Dominós D-48 & 16 & 45,7 & Becasse Atitudes Sócio Emocionais Crianças Pré-escolares & 4 & 11,4 \\
\hline Escala de Inteligência Wechsler Ciranças & 16 & 45,7 & Bateria Burocrática VIG & 3 & 8,6 \\
\hline O desenho da figura humana & 16 & 45,7 & BBT & 3 & 8,6 \\
\hline Teste de Inteligência Não Verbal G38 & 15 & 42,9 & Cornell Index & 3 & 8,6 \\
\hline Matrizes Progressivas Coloridas & 15 & 42,9 & Destreza Digital & 3 & 8,6 \\
\hline Inventário de Sintomas de Stress para Adultos Lipp & 14 & 40 & Escala de Personalidade de Comrey & 3 & 8,6 \\
\hline Inv. Mult. Minnesota Pers. MMPI & 14 & 40 & Escala de Sociabilidade e Emotividade ESE & 3 & 8,6 \\
\hline Matrizes Progressivas - escala Avançada & 14 & 40 & Inventário de Administração de Tempo ADT & 3 & 8,6 \\
\hline Matrizes Progressivas - escala geral & 14 & 40 & Medida de Fluência Verbal & 3 & 8,6 \\
\hline Teste das Pirâmides Coloridas & 14 & 40 & Teste Equicultual Inteligência & 3 & 8,6 \\
\hline Teste Wartegg & 14 & 40 & BFM Bateria de Funções Mentais para Morotistas & 3 & 8,6 \\
\hline Teste Psicodiagnóstico Miocinético PMK & 14 & 40 & D2 Teste de Atenção Concentrada & 3 & 8,6 \\
\hline Inventário Interesses Angelini e Thustone & 14 & 40 & Escala Avaliação do Comportamento Infantil Para Professor & 3 & 8,6 \\
\hline INV & 13 & 37,1 & Teste de Frases Incompletas FIGS & 3 & 8,6 \\
\hline Supl. Teste Apercepção Infantil CAT-S & 13 & 37,1 & Avaliação da Criatividade por figuras e palavras & 3 & 8,6 \\
\hline Teste de Aptidões Específicas DAT & 13 & 37,1 & Bateria TSP & 2 & 5,7 \\
\hline Bender Infantil & 12 & 34,3 & Diagnóstico Tipológico Organizacional - DTO & 2 & 5,7 \\
\hline Kuder Inv. Interesses Profissionais & 12 & 34,3 & Inventário STAXI & 2 & 5,7 \\
\hline Bateria de Testes de Aptidão BTAG & 11 & 31,4 & Inventário Expectativas e Crenças IECPA & 2 & 5,7 \\
\hline O Teste Gestáltico Bender para Crianças & 11 & 31,4 & IAR Inst. Aval. Repert. Básico Alfab. & 2 & 5,7 \\
\hline Teste das Pirâmides das cores 24M Pfister & 11 & 31,4 & Liderança e Poder & 2 & 5,7 \\
\hline Atenção Concentrada & 10 & 28,6 & Memória $\mathrm{R}$ & 2 & 5,7 \\
\hline BPR-5 & 10 & 28,6 & Prova de Nível Mental & 2 & 5,7 \\
\hline Inventário de Ansiedade IDATE & 10 & 28,6 & Questionário Personalidade Dadahie & 2 & 5,7 \\
\hline LIP Levantamento Interesses Profissionais & 10 & 28,6 & Questionário do Adolescente R-4 & 2 & 5,7 \\
\hline O Teste das pirâmides das cores & 10 & 28,6 & Questionário Intimo & 2 & 5,7 \\
\hline Inventário de Habilidades Sociais & 10 & 28,6 & Reprodução de Figuras & 2 & 5,7 \\
\hline Escala de Stress Infantil ESI & 9 & 25,7 & Teste Caracterológico & 2 & 5,7 \\
\hline Inventário Fatorial de Personalidade & 9 & 25,7 & Teste de Atenção Difusa & 2 & 5,7 \\
\hline Teste Metropolitano & 9 & 25,7 & Teste de Capacidades Intelectuais TCI & 2 & 5,7 \\
\hline Teste Não verbal de Inteligência R-1 & 9 & 25,7 & Teste de Frustração & 2 & 5,7 \\
\hline Teste Palográfico & 9 & 25,7 & Teste de Liderança Situacional TLS & 2 & 5,7 \\
\hline Inventário de Interesses Profissionais & 8 & 22,9 & Teste Organização Percepto Motora TOP & 2 & 5,7 \\
\hline Atenção Concentrada 15 & 7 & 20 & Teste Prontidão Horizontes & 2 & 5,7 \\
\hline Becasse Maturidade Escolar & 7 & 20 & Escala de Preconceito Profissional EPP & 1 & 2,9 \\
\hline Escala de Transtorno de Déficit de Atenção/Hiperatividade & 7 & 20 & Mandala de Palavras & 1 & 2,9 \\
\hline Figuras Complexas de Rey & 7 & 20 & O desenvolv. Comp, Criança Primeiro ano & 1 & 2,9 \\
\hline Inventário de Ansiedade IDATE-C & 7 & 20 & Questionário Confidencial & 1 & 2,9 \\
\hline Programação Hábitos e Desempenho PHD & 7 & 20 & Sondagem de Habilidade & 1 & 2,9 \\
\hline Quati Questionário Avaliação Tipológica & 7 & 20 & Teste de Agradabilidade Básica & 1 & 2,9 \\
\hline Teste de Desempenho Escolar TDE & 7 & 20 & Teste de catálogo Livros Bessa Tramer & 1 & 2,9 \\
\hline Teste D-70 & 7 & 20 & Teste de Conceitos Básicos de Bohen & 1 & 2,9 \\
\hline Teste Raven Operações Lógicas RTLO & 7 & 20 & Teste de Estruturas Vocacionais TEV & 1 & 2,9 \\
\hline Becasse Maturidade Escolar & 7 & 20 & Teste Raciocínio Lógico-Numérico & 1 & 2,9 \\
\hline Escala de Stress Infantil ESI & 7 & 20 & Teste de Sondagem Intelectual & 1 & 2,9 \\
\hline Coleção Papel de Carta & 6 & 17,1 & Teste Desenho Silver Coginição e emoção & 1 & 2,9 \\
\hline Como Chefiar? & 6 & 17,1 & Teste Edites de Inteligência TEI & 1 & 2,9 \\
\hline Inventário de Atitudes de Trabalho IAT & 6 & 17,1 & Teste Projetivo Omega & 1 & 2,9 \\
\hline Lista de Problemas Pessoais Adultos & 6 & 17,1 & Teste Projetivo Sonoro & 1 & 2,9 \\
\hline Lista de Problemas Pessoais adolescentes & 6 & 17,1 & ACRE Teste Atenção Concentrada e Rapidez e Exatidão & 1 & 2,9 \\
\hline QVI Questionário Vocacional Interesses & 6 & 17,1 & Coordenação Bi-manual Edites & 1 & 2,9 \\
\hline Teste Coletivo Inteligência Adultos - CIA & 6 & 17,1 & MM Teste a minhas mãos & 1 & 2,9 \\
\hline Teste das Cores & 6 & 17,1 & R-6 Avaliação de Chefia & 1 & 2,9 \\
\hline BTN Bateria de Testes Neuropsicológicos & 6 & 17,1 & TAA Teste de Aptidão Acadêmica & 1 & 2,9 \\
\hline R2 Teste não verbal de Inteligência & 6 & 17,1 & Teste das Dinâmicas Profissionais TDP & 1 & 2,9 \\
\hline Escala Reduzida de Autoconceito ERA & 5 & 14,3 & Teste de Aptidão a Mecânica TAM & 1 & 2,9 \\
\hline Teste Barcelona & 5 & 14,3 & DDTVP-2 Teste Evolutivo de Percepção Visual & 1 & 2,9 \\
\hline Teste dos Relógios & 5 & 14,3 & BFM 1 & 1 & 2,9 \\
\hline Teste de prontidão para leitura & 5 & 14,3 & BFM 2 & 1 & 2,9 \\
\hline Escala déficit Atenção Hiperatividade & 5 & 14,3 & Escala fatorial de Ajustamento Emocional/Neuroticismo & 1 & 2,9 \\
\hline
\end{tabular}


Tabela 4. Distribuição das Freqüências e Porcentagens Referentes aos Instrumentos Psicológicos mais Utilizados por Psicólogos Comportamentais ( $n=35)$ (Continuação)

\begin{tabular}{|c|c|c|c|c|c|}
\hline Testes Mais conhecidos & $\mathbf{F}$ & $\%$ & Testes Mais conhecidos & $\boldsymbol{F}$ & $\%$ \\
\hline Teste de Apercepção Infantil CAT-H & 20 & 57,1 & Escala Reduzida de Autoconceito ERA & 3 & 8,6 \\
\hline Bender Infantil & 18 & 51,4 & Escala de Escolha Profissional & 3 & 8,6 \\
\hline O desenho da figura humana & 14 & 40 & Diagnóstico Organizacional & 3 & 8,6 \\
\hline Atenção Concentrada & 10 & 28,6 & Escala déficit Atenção Hiperatividade & 3 & 8,6 \\
\hline O Teste Gestaltico Bender para Crianças & 9 & 25,7 & Teste Zulliger & 3 & 8,6 \\
\hline Teste Wartegg & 9 & 25,7 & Como Chefiar? & 3 & 8,6 \\
\hline Inventário de Interesses Profissionais & 8 & 22,9 & Teste D-70 & 3 & 8,6 \\
\hline Escala de Maturidade Mental Columbia & 8 & 22,9 & Becasse Maturidade Escolar & 2 & 5,7 \\
\hline Escala de Inteligência Wechsler Crianças & 8 & 22,9 & Teste de Atenção Difusa & 2 & 5,7 \\
\hline Inventário de Habilidades Sociais & 8 & 22,9 & Questionário do Adolescente R-4 & 2 & 5,7 \\
\hline LIP Levantamento Interesses Profissionais & 7 & 20 & Quati Questionário Avaliação Tipológica & 2 & 5,7 \\
\hline Dezesseis PF & 7 & 20 & Prova de Nível Mental & 2 & 5,7 \\
\hline Inventário Interesses Angelini e Thustone & 7 & 20 & Lista de Problemas Pessoais adolescentes & 2 & 5,7 \\
\hline Bateria CEPA & 6 & 17,1 & Inventário de Atitudes de Trabalho IAT & 2 & 5,7 \\
\hline Matrizes Progressivas - escala geral & 6 & 17,1 & Figuras Complexas de Rey & 2 & 5,7 \\
\hline BPR-5 & 6 & 17,1 & Atenção Concentrada 15 & 2 & 5,7 \\
\hline Escala de Stress Infantil ESI & 6 & 17,1 & Escala de Stress Infantil ESI & 2 & 5,7 \\
\hline WISC III & 6 & 17,1 & Escala Avaliação do Comportamento Infantil Para Professor & 2 & 5,7 \\
\hline Teste de Rorschach & 6 & 17,1 & Teste Prontidão Horizontes & 2 & 5,7 \\
\hline Teste de Apercepção Infantil CAT-A & 5 & 14,3 & Teste Não verbal de Inteligência R-1 & 2 & 5,7 \\
\hline INV & 5 & 14,3 & Teste Organização Percepto Motora TOP & 1 & 2,9 \\
\hline Matrizes Progressivas - escala Avançada & 5 & 14,3 & Teste de Liderança Situacional TLS & 1 & 2,9 \\
\hline Inv. Mult. Minnesota Pers. MMPI & 5 & 14,3 & Teste Habilidade Trabalho Mental HTM & 1 & 2,9 \\
\hline Inventário de Sintomas de Stress para Adultos Lipp & 5 & 14,3 & Teste de Estruturas Vocacionais TEV & 1 & 2,9 \\
\hline Escala de Transtorno de Déficit de Atenção/Hiperatividade & 5 & 14,3 & Teste de Aptidões Específicas DAT & 1 & 2,9 \\
\hline Teste Psicodiagnóstico Miocinético PMK & 5 & 14,3 & Teste das Pirâmides Coloridas & 1 & 2,9 \\
\hline Teste de Desempenho Escolar TDE & 4 & 11,4 & Teste das Cores & 1 & 2,9 \\
\hline Inventário Fatorial de Personalidade & 4 & 11,4 & Teste Coletivo Inteligência Adultos - CIA & 1 & 2,9 \\
\hline Inventário de Ansiedade IDATE-C & 4 & 11,4 & Questionário Saúde Geral Goldberg QSG & 1 & 2,9 \\
\hline Inventário de Ansiedade IDATE & 4 & 11,4 & Questionário Personalidade Dadahie & 1 & 2,9 \\
\hline Teste de Inteligência Não Verbal G38 & 4 & 11,4 & Programação Hábitos e Desempenho PHD & 1 & 2,9 \\
\hline Teste de Inteligência Não Verbal G36 & 4 & 11,4 & Medida de Fluência Verbal & 1 & 2,9 \\
\hline Dominós D-48 & 4 & 11,4 & Escala de Personalidade de Comrey & 1 & 2,9 \\
\hline Teste Palográfico & 4 & 11,4 & Diagnóstico Tipológico Orgnazacional - DTO & 1 & 2,9 \\
\hline Teste Metropolitano & 4 & 11,4 & R2 Teste não verbal de Inteligência & 1 & 2,9 \\
\hline Teste de Apercepção Temática TAT & 3 & 88,6 & Escala fatorial de Ajustamento Emocional/Neuroticismo & 1 & 2,9 \\
\hline Teste das Fábulas & 3 & 8,6 & Avaliação da Criatividade por figuras e palavras & 1 & 2,9 \\
\hline Supl. Teste Apercepção Infantil CAT-S & 3 & 8,6 & Inventário e Auto-análise dos Interesses Prof. IAIP & 1 & 2,9 \\
\hline QVI Questionário Vocacional Interesses & 3 & 8,6 & Teste das Pirâmides das cores 24M Pfister & 1 & 2,9 \\
\hline O Teste das pirâmides das cores & 3 & 8,6 & Questionário Desiderativo & 1 & 2,9 \\
\hline Matrizes Progressivas Coloridas & 3 & 8,6 & Lendo e Escrevendo & 1 & 2,9 \\
\hline Lista de Problemas Pessoais Adultos & 3 & 8,6 & Teste de prontidão para leitura & 1 & 2,9 \\
\hline IAR Inst. Aval. Repert. Básico Alfab. & 3 & 8,6 & & & \\
\hline
\end{tabular}

\section{DISCUSSÃO}

Constatou-se que a avaliação é utilizada pela maior parte dos profissionais com a finalidade de diagnóstico e ao final de cada intervenção servindo de base para avaliação da prática do profissional. Dado similar foi observado na pesquisa de Oliveira, Noronha, Beraldo e Santarem (2003) com estagiários de clínica comportamental.

Esse resultado, de certo modo, é característico do psicólogo comportamental/cognitivo, visto que não fecha o diagnóstico inicialmente (Matos, 1999), mas a cada intervenção os objetivos e as hipóteses de avaliação são revistos e novamente avaliados para uma intervenção futura. Esse dado vem corroborar o que afirmam Godoy (1996) e Lettner (1998), ou seja, que a avaliação comportamental é um processo contínuo de testagem de hipóteses que vai desde o diagnóstico até a avaliação dos efeitos provocados pela intervenção realizada. Nesse sentido, pode-se dizer que o psicólogo comportamental está em constante processo de avaliação.

As entrevistas são as estratégias/instrumentos utilizadas com maior freqüência na avaliação. Tanto a avaliação qualitativa quanto a quantitativa são realizadas pelos profissionais e a alternativa "sempre" foi a mais pontuada para as duas alternativas. Desse modo, os resultados apontam que os profissionais recorrem à avaliação na sua prática. Esse fato é muito importante, pois tomar decisões sem avaliar não é algo esperado de um bom profissional.

No que se refere à atuação profissional dos psicólogos, foi evidenciado que a maioria atua em 
consultórios particulares ou em universidades e a abordagem que norteia a prática da maioria é a comportamental/cognitiva. Evidenciou-se, ainda, que a maior parte dos profissionais possui mestrado ou doutorado, ou são especialistas. Trata-se, portanto, de profissionais que possuem um conhecimento maior em relação àqueles que somente apresentam a graduação em psicologia.

Desse modo, poder-se-ia supor que tais profissionais deveriam seguir o que preconizam os pressupostos da avaliação comportamental, que, segundo Haynes e O' Brien (2000), são características acentuadamente divergentes em relação à avaliação tradicional. A ênfase estaria no estabelecimento de relações funcionais ao invés de causais, nas quais o comportamento-problema seria entendido com base nos princípios da aprendizagem social e da análise experimental do comportamento, e não na teoria tradicional da personalidade.

Não obstante, os resultados evidenciaram que não há consonância entre a abordagem teórica desses profissionais e a utilização de instrumentos psicológicos, tendo-se em vista que, surpreendentemente, os três testes mais utilizados pelos psicólogos comportamentais desta pesquisa são testes cuja fundamentação está na teoria tradicional da personalidade (Teste de A percepção Infantil CAT-H, Bender Infantil e $\mathrm{O}$ desenho da figura humana). A falta de coerência entre a abordagem teórica adotada (comportamental/cognitiva) e a utilização de instrumentos psicológicos (psicodinâmicos) já foi observada em pesquisas anteriores realizadas por Watkins, Campbell e McGregor (1990) e Oliveira e cols. (2003).

Um psicólogo comportamental pode utilizar testes e inventários em sua avaliação, contudo tais instrumentos são auxiliares na avaliação e têm por base a teoria psicométrica de construção de instrumento (Haynes \& O'Brien, 2000; Meyer, 2001; Piotrowski \& Lubin, 1990). Não há como ignorar que existe divergência entre a teoria e a prática profissional, mas utilizar instrumentos não concernentes à teoria comportamental/cognitiva é algo que realmente causa estranheza. Não se sabe se essa falta de consonância está associada a uma formação falha ou a uma prática inadequada, contudo é sabido que esse fato não pode mais ser ignorado.

\section{CONSIDERAÇÕES FINAIS}

O presente estudo teve como objetivo a caracterização das estratégias, das técnicas e dos instrumentos psicológicos mais conhecidos e utilizados por profissionais, com abordagem comportamental ou cognitiva Os resultados do presente estudo, pouco animadores, revelam a incoerência existente na prática profissional do psicólogo brasileiro, uma vez que a maioria dos participantes faz uso de técnicas projetivas como instrumento de coleta de dados quando do processo de avaliação psicológica.

Os achados estão em concordância com a pesquisa desenvolvida por Oliveira e cols. (2003), cujo objetivo se assemelhou ao do presente trabalho, embora tenha sido realizado com uma amostra de estudantes. Os resultados evidenciaram que os participantes utilizavam inventários de personalidade e técnicas gráficas projetivas para compor sua avaliação, o que revela a falta de compreensão de princípios básicos da testagem psicológica.

Vale ressaltar que instrumentos de medida são construídos à luz de uma teoria psicológica e que seus resultados serão analisados tendo essa teoria como referência. Em vista disso, parece ficar claro que os instrumentos projetivos têm como base as teorias psicanalíticas, que, por sua vez, em muito se diferenciam dos conceitos anunciados pela abordagem comportamental/cognitiva. Causa-nos estranheza que profissionais possam fazer uso de um instrumento sem comungar, ou mais enfaticamente, sendo contrário aos pressupostos teóricos usados em sua construção. Diante do exposto, restam duas questões, a saber: os psicólogos desconhecem as teorias de construção de recursos de avaliação ou não compreendem que há uma extensa incoerência em sua atuação profissional.

\section{REFERÊNCIAS}

Almeida, L. S. (1999). Avaliação psicológica: exigências e desenvolvimento nos seus métodos. Em S. M. Wechsler \& R. S. L. Guzzo (Orgs.), Avaliação psicológica: perspectiva internacional (pp. 41-55). São Paulo: Casa do Psicólogo.

American Educational Research Association, American Psychological Association \& National Council on Measurement in Education (1999). Standards for Educational and Psychological Testing. Washington: American Educational Research Association.

Anastasi, A. \& Urbina, S. (2000). Fundamentos da testagem psicológica. Porto Alegre: Artes Médicas.

Casullo, M. M. (1999). Evaluacion psicológica. Em S. M. Wechsler \& R. S. L. Guzzo (Orgs.), Avaliação psicológica: perspectiva internacional (pp. 23-39). São Paulo: Casa do Psicólogo.

Cunha, A. C. B. (1998). Análise experimental do comportamento. Em B. Rangé (Org.), Psicoterapia 
comportamental e cognitiva: pesquisa, prática, aplicações e problemas (pp.15-25). Campinas: Psy.

Godoy, A. (1996). O processo da avaliação comportamental. Em V. E. Caballo (Org.), (M. D. Claudino, Trad.) Manual de técnicas de terapia comportamental e modificação do comportamento (pp. 8197). São Paulo: Livraria Santos.

Haynes, S. H. \& O' Brien, W. H. (2000). Background, characteristics and history. Em S. N. Haynes \& W. H. O'Brien (Orgs.), Principles and practice of behavioral assessment (pp.265-292). New York: Kluwer Academic/Plenum Publishers.

International Test Commition - ITC (2001). Diretrices internacionales para el uso de los tests. Disponível em: <www.cop.es/tests>. (Acessado em 04/03/2001).

Lettner, H. W. (1998). Avaliação comportamental. Em B. Rangé (Org.), Psicoterapia comportamental e cognitiva de transtornos psiquiátricos (pp. 27-31). Campinas: Psy.

Matos, M. A. (1998). Contingências para análise comportamental no Brasil. Revista Psicologia USP, 9(1), 89-100.

Matos, M. A. (1999). Análise funcional do comportamento. Estudos de Psicologia, 6(3), 8-18.

Meyer, S. B. (2001, $2^{\mathrm{a}}$ ed. rev.). O conceito de análise funcional. Em M. Delitti (Org.), Sobre comportamento e cognição: a prática da análise do comportamento e da terapia cognitivo-comportamental (pp. 29-34). Santo André: ESETec.

Noronha, A. P. P. (1999). Avaliação Psicológica: usos e problemas com ênfase nos testes. Tese de Doutorado. Instituto de Psicologia e Fonoaudiologia da Pontifícia Universidade Católica de Campinas, Campinas.
Noronha, A. P. P., Primi, R. \& Alchieri, J. C. (2002). Parâmetros psicométricos: uma análise de testes psicológicos comercializados no Brasil. Manuscrito submetido à Revista Psicologia: ciência e profissão.

Oliveira, K. L., Noronha, A. P. P., Beraldo, F. N. M. B. \& Satarem, E. M. (2003). Utilização de técnicas e instrumentos psicológicos: uma pesquisa com estagiários de clínica comportamental. Psico-PUCRS, 34(1), 123-140.

Pasquali, L. (Org.) (1999). Instrumentos psicológicos: manual prático de elaboração. Brasília: LabPAM; IBAP.

Pasquali, L. (Org.) (2001). Técnicas do Exame Psicológico - TEP. Manual: Fundamentos das Técnicas Psicológicas (V.1). São Paulo: Casa do Psicólogo.

Piotrowski, C. \& Lubin, B. (1990). Assessment pratices of health psychologist: survey of APA Division $38^{\mathrm{a}}$ Clinicians. Professional Psychology: Research and Practice, 21(2), 99-106.

Rangé, B. (1998). Psicoterapia Comportamental. Em B. Rangé (Org.), Psicoterapia comportamental e cognitiva: pesquisa, prática, aplicações e problemas (pp. 35-42). Campinas: Psy.

Watkins, C. E. Jr., Campbell, V. L. \& McGregor, P. (1990). What types of psychological tests do Behavioral (and other) conunseling psychologists use? The Behavior Therapist, 13, 115-117.

Recebido em 18/12/2003 Aceito em 30/05/2004

Endereço para correspondência: Katya Luciane de Oliveira. Rua Maestro Sebastião Peranovich, 415, Atibaia Jardim, CEP 12940-000, Atibaia-SP. E-mail: katya_lincoln@ig.com.br 